\title{
Single-cell and spatial transcriptomics approaches of cardiovascular development and disease
}

\author{
Robert Roth ${ }^{1}$, Soochi Kim ${ }^{2}$, Jeesu Kim ${ }^{3}$ \& Siyeon Rhee, \\ ${ }^{1}$ Department of Biology, Stanford University, Stanford, CA 94305, ${ }^{2}$ Department of Neurology and Neurological Sciences, Stanford \\ University School of Medicine, Stanford, CA 94304, USA, ${ }^{3}$ Department of Cogno-Mechatronics Engineering, Pusan National University, \\ Busan 46241, Korea
}

Recent advancements in the resolution and throughput of single-cell analyses, including single-cell RNA sequencing (scRNA-seq), have achieved significant progress in biomedical research in the last decade. These techniques have been used to understand cellular heterogeneity by identifying many rare and novel cell types and characterizing subpopulations of cells that make up organs and tissues. Analysis across various datasets can elucidate temporal patterning in gene expression and developmental cues and is also employed to examine the response of cells to acute injury, damage, or disruption. Specifically, scRNA-seq and spatially resolved transcriptomics have been used to describe the identity of novel or rare cell subpopulations and transcriptional variations that are related to normal and pathological conditions in mammalian models and human tissues. These applications have critically contributed to advance basic cardiovascular research in the past decade by identifying novel cell types implicated in development and disease. In this review, we describe current scRNA-seq technologies and how current scRNA-seq and spatial transcriptomic (ST) techniques have advanced our understanding of cardiovascular development and disease. [BMB Reports 2020; 53(8): 393-399]

\section{INTRODUCTION}

Within the field of cardiovascular biology, there have been many questions about understanding how the heart develops and how developmental cues go awry in the disease progression of congenital disorders. The mammalian heart contains many specialized cell types with differences in morphology, location, gene expression patterns, and functions. These heterogenous cell types that comprise of the heart have been inves-

*Corresponding author. Tel: +1-650-725-4816; Fax: +1-650-7236132; E-mail: syr@stanford.edu

https://doi.org/10.5483/BMBRep.2020.53.8.130

Received 25 May 2020

Keywords: Cardiovascular development, Cardiovascular disease, Single-cell RNA sequencing, Spatial transcriptomics tigated at the cellular and molecular levels. However, a major drawback of these techniques is in measuring the average expression or patterns across the samples examined, resulting in the loss individual of cellular and molecular resolutions. To better understand cardiovascular development and disease, resolving the subtle differences between heterogeneous cardiac cells may have profound therapeutic implications.

scRNA-seq technologies have achieved significant progress in the last several years and have become one of the most popular tools for understanding cell heterogeneity and lineage progression (1-3). Tang et al. in 2009 first reported whole-transcriptome sequencing in single cells. Although technologies have advanced in throughput and resolution, the methodology of the pipeline remains similar to current techniques (4).

The scRNA-seq method involves dissociation of a tissue, which requires breaking the connection between single cells by breaking down extracellular connections, leading to a loss of individual cell localization information and can cause incomplete interpretation. Presently, there are two main scRNA-seq approaches: SMART-seq2 and 10x genomics. SMART-seq2 is used to profile the full length of transcripts but provides a relatively low throughput; 10x genomics can capture more cells but has limited ability to capture transcripts (5-8). Besides these scRNA-seq techniques, spatially resolved transcriptomics and in situ sequencing (ISS) also have made important progress (9, 10). Also, ISS, which achieves cell-level gene mapping in single cells in situ, can be used for cell-level mapping of organ development by a fluorescent-imaging-based sequencing method (Fig. 1) (11-13).

By means of scRNA-seq, researchers can map expression profiles at single-cell resolution. Since the introduction of scRNAseq and ST, the number of single-cell experiments has greatly increased. The scRNA-seq technique has become a powerful tool for identifying and classifying cell subpopulations, characterizing rare or small subpopulations, and tracing cells along dynamic cellular stages, such as during differentiation, which has combined to understanding cardiovascular development and pathogenesis $(3,11,14,15)$. In this review, we briefly describe the history and progression of scRNA-seq and ST technologies, and summarize the recent scRNA-seq studies in cardiovascular research and their potential implications for understanding the

ISSN: 1976-670X (electronic edition)

Copyright (C) 2020 by the The Korean Society for Biochemistry and Molecular Biology

(c) This is an open-access article distributed under the terms of the Creative Commons Attribution Non-Commercial License (http://creativecommons.org/licenses/by-nc/4.0) which permits unrestricted non-commercial use, distribution, and reproduction in any medium, provided the original work is properly cited. 


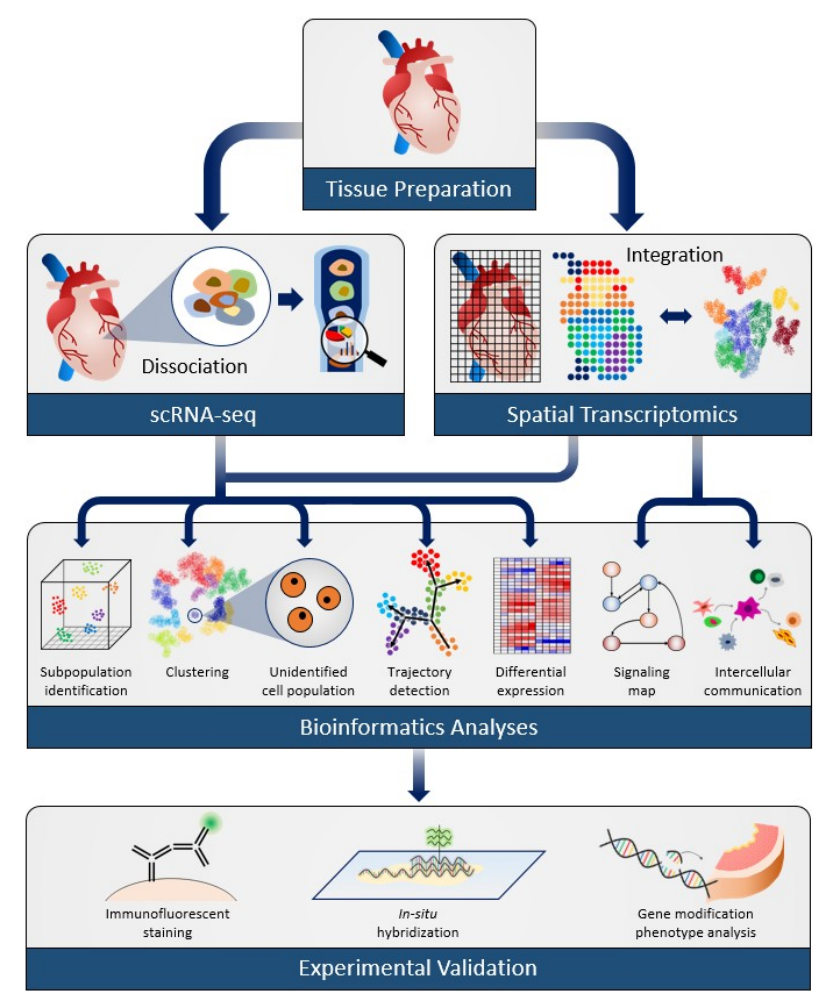

Fig. 1. A framework of scRNA-seq and ST applications in cardiovascular research.

cardiovascular system and associated diseases (16).

\section{HISTORY AND DEVELOPMENT OF SINGLE-CELL-RNA AND TRANSCRIPTOMICS SEQUENCING ANALYSIS}

Recent advances in biotechnology, specifically single-cell technologies, have revolutionized basic science research. In 1992, Eberwine et al. developed the T7 amplification technique, which can increase amounts of RNA from low concentration of starting material, and assessed expression profiles in live pyramidal neurons of the rat hippocampus (17). However, its application to molecular and cellular biology was limited to detecting only a few genes of interest by probes or RT-PCR, until the introduction of next-generation sequencing platforms. The complexity of the transcriptomes of single cells was first reported in 2009, using an mRNA-Seq assay (4). This new technique was applied to a single mouse blastomere to profile its digital gene expression and identified 1,753 previously unknown splice junctions at single-cell resolution. Since then, advances in scRNA-seq and downstream analysis tools have greatly accelerated our understanding of the complexity of gene expression, regulation, and networks.

More recently, the Chan Zuckerberg Biohub released Tabula Muris, a compendium of scRNA-seq data from 20 mouse organs and tissues (18). The data provide references to cell-type composition within and between organs and tissues and identified rare or poorly characterized cell populations along with their gene expression patterns. These data represent a new resource for both cell-centric and gene-centric studies across 20 tissues. The cited study used two distinct technical approaches for most organs: 3 '-end counting based on microfluidic droplets and full-length transcript analysis based on fluorescenceactivated cell sorting (FACS). The differences in technical approaches will be discussed in more detail in the following section.

Detecting gene expression in single cells is challenging, as the concentration of total RNA present in a cell is on the order of picograms, and of those, polyadenylated mRNA constitutes less than $5 \%$ of total cellular RNA $(19,20)$. In the last decades, different high-throughput methods for scRNA-seq have been developed to isolate and capture these finite biomolecules efficiently, with different degrees of coverage, sensitivity, and multiplexing capabilities for single-cell transcriptome analysis. The key technological developments and protocol improvements that enabled large-scale scRNA-seq experiments have been previously reviewed by Svensson et al. (21).

Current scRNA-seq methods can be classified based on the single-cell isolation techniques and capture methods for library preparation (Table 1). Three platforms are available for sample preparation, plate-based, microfluidics, and droplets. Of those, the two most-popular approaches are plate-based SMART-seq in the Fluidigm C1 system and microdroplet-based scRNA-seq from 10X Chromium. SMART-seq2 is an improved version of SMART-seq, which relies on template switching and preamplification, with an improvement in both the cDNA library yield and average length from individual cells (22). This method has several tradeoffs, but makes possible the analysis of all exons of each transcript and detection of the different splice variants. SMART-seq2 also enables comprehensive SNP and mutation analysis, widening its field of application (23). Limitations of this approach include refining the selectivity for polyadenylated RNA, precluding analysis of polyA-RNA that does not reflect the strand-specific nature of mRNAs. In addition, this method relies on the labor of individual researchers rather than automated methods of multiplexing $(19,23)$.

STRT was one of the early multiplexing methods developed, although it precludes splice isoforms. This method reflects the strand-specific nature of mRNAs and identifies the transcription start site (TSS) (19). CEL-seq is another early method that implemented barcoding and pooling samples, but was the first to use linear in vitro transcription (IVT) amplification (24). Hashimshony et al. reported that CEL-seq outperforms STRT in terms of reproducibility, sensitivity, and robustness. CEL-seq does not detect miRNAs and other non-polyadenylated transcripts, which can be its strength, because the barcoded transcripts preclude rRNA, which increases the efficiency of the sequenced reads. Both methods are limited to sequencing of the different ends of the mRNA transcript ( $3^{\prime}$ bias for CEL-seq and $5^{\prime}$ bias for STRT), which severely limits their 
Table 1. Summary of scRNA-seq techniques

\begin{tabular}{|c|c|c|c|c|c|c|c|c|}
\hline Techniques & $\begin{array}{l}\text { Single cell } \\
\text { isolation } \\
\text { techniques }\end{array}$ & Capture Platforms & $\begin{array}{l}\text { cDNA } \\
\text { ampl. }\end{array}$ & $\begin{array}{l}\text { Strand } \\
\text { specific }\end{array}$ & $\begin{array}{l}\text { Multi- } \\
\text { plexing }\end{array}$ & UMI (bp) & Features & Year / (Refs) \\
\hline \multicolumn{9}{|c|}{ Full-length transcript } \\
\hline Tang method & Manual & PCR tubes & PCR & $x$ & $x$ & & $\begin{array}{l}\text { Splice variant } \\
\text { detection }\end{array}$ & $2009(4)$ \\
\hline SMART-seq & FACS & Plate based & PCR & $x$ & $x$ & & $\begin{array}{l}\text { Template } \\
\text { switching reaction }\end{array}$ & $2012(7)$ \\
\hline SMART-seq2 & FACS & Plate based & PCR & $x$ & $x$ & & $\begin{array}{l}\text { Improved yield, } \\
\text { coverage and } \\
\text { accruacy }\end{array}$ & $2013(22)$ \\
\hline Quartz-seq & FACS & Plate based & PCR & $x$ & $x$ & & $\begin{array}{l}\text { Single PCR } \\
\text { tube reaction } \\
\text { without } \\
\text { any purification }\end{array}$ & $2013(8)$ \\
\hline MATQ-seq & FACS & Plate based & PCR & $\mathrm{O}$ & $\mathrm{O}$ & & $\begin{array}{l}\text { Whole gene body } \\
\text { coverage }\end{array}$ & $2017(20)$ \\
\hline \multicolumn{9}{|l|}{ 5' End transcript } \\
\hline STRT & FACS & Plate based & PCR & $\mathrm{O}$ & $x$ & & $\begin{array}{l}\text { Highly multiplexed, } \\
\text { TSS detection }\end{array}$ & $2012(17)$ \\
\hline STRT-seq-2i & $\begin{array}{l}\text { Limiting dilution or } \\
\text { FACS }\end{array}$ & $\begin{array}{l}\text { Microarray platform } \\
\text { custom capture } \\
\text { plates }\end{array}$ & PCR & $\mathrm{O}$ & $\mathrm{O}$ & 6 & $\begin{array}{l}\text { Imaging checkpoint } \\
\text { available }\end{array}$ & $2017(26)$ \\
\hline WaferGen & $\begin{array}{l}\text { Multi-sample } \\
\text { nanodispenser } \\
\text { (MSND) }\end{array}$ & $\begin{array}{l}\text { Nanowell based } \\
\text { (ICELL8) }\end{array}$ & PCR & $\mathrm{O}$ & $\mathrm{O}$ & 10 & $\begin{array}{l}\text { Imaging software to } \\
\text { identify nanowells }\end{array}$ & $2017(29)$ \\
\hline \multicolumn{9}{|l|}{$3^{\prime}$ End transcript } \\
\hline CEL-seq & FACS & Plate based & IVT & $\mathrm{O}$ & $x$ & & $\begin{array}{l}\text { First to use linear } \\
\text { RNA amplification }\end{array}$ & $2012(22)$ \\
\hline MARS-seq & Microfluidic, FACS & Plate based & IVT & $\mathrm{O}$ & $\mathrm{O}$ & 4 & $\begin{array}{l}\text { Introduced } \\
\text { automated } \\
\text { massively }\end{array}$ & $2014(23)$ \\
\hline CytoSeq & $\begin{array}{l}\text { Recursive } \\
\text { Poision loading }\end{array}$ & Nanowell & PCR & $\mathrm{O}$ & $\mathrm{O}$ & 8 & $\begin{array}{l}\text { First to present } \\
\text { scalable } \\
\text { mRNA cytometry }\end{array}$ & 2015 (34) \\
\hline Drop-seq & $\begin{array}{l}\text { Double } \\
\text { poisson loading, } \\
\text { Microfluidic } \\
\text { channel }\end{array}$ & Droplet based & PCR & $\mathrm{O}$ & $\mathrm{O}$ & 8 & $\begin{array}{l}\text { Coencapsulation of } \\
\text { single cells }\end{array}$ & 2015 (29) \\
\hline InDrop & $\begin{array}{l}\text { Super } \\
\text { Poisson loading }\end{array}$ & Droplet based & IVT & $\mathrm{O}$ & $\mathrm{O}$ & 6 & $\begin{array}{l}\text { Cells are captured } \\
\text { and barcoded }\end{array}$ & $2015(27)$ \\
\hline CEL-seq2 & $\begin{array}{l}\text { Manual, } \\
\text { Microfluidic, } \\
\text { FACS, C1 }\end{array}$ & Microfluidic chip & IVT & $\mathrm{O}$ & $\mathrm{O}$ & 6 & $\begin{array}{l}\text { Implemented } \\
\text { the UMI }\end{array}$ & $2016(24)$ \\
\hline DroNC-seq & Poisson loading & Droplet based & PCR & $\mathrm{O}$ & $\mathrm{O}$ & 8 & High throughput & 2017 (32) \\
\hline Chromium 10x & $\begin{array}{l}\text { Super } \\
\text { Poisson loading }\end{array}$ & $\begin{array}{l}\text { Droplet based } \\
\text { (Single Cell A } \\
\text { Chip) }\end{array}$ & PCR & $\mathrm{O}$ & $\mathrm{O}$ & 10 & $\begin{array}{l}\text { High throughput, } \\
\text { process up }\end{array}$ & 2017 (33) \\
\hline SPLiT-seq & $\begin{array}{l}\text { No partitioning into } \\
\text { compartments }\end{array}$ & Plate based & PCR & $\mathrm{O}$ & $\mathrm{O}$ & & $\begin{array}{l}\text { Can sequence fixed } \\
\text { cells or nuclei }\end{array}$ & $2018(36)$ \\
\hline Quartz-seq2 & FACS & Plate based & PCR & $\mathrm{O}$ & $\mathrm{O}$ & 8 & $\begin{array}{l}\text { Major improvement } \\
\text { of Poly(A)tagging }\end{array}$ & $2018(30)$ \\
\hline MARS-seq2 & FACS & Plate based & IVT & $\mathrm{O}$ & $\mathrm{O}$ & 8 & $\begin{array}{l}\text { Integrated pipelines } \\
\text { for index sorting }\end{array}$ & 2019 (35) \\
\hline
\end{tabular}


ability to distinguish splice isoforms $(19,24)$. CEL-seq2 is a modified version of CEL-seq. CEL-seq2 is compatible with different platforms and is the first technique to implement a single-cell, on-chip barcoding method on the Fluidigm's C1 system. CEL-seq2 outperforms both its initial version and SMART-seq on the C1 system (25). A modified and extensively reoptimized version of the 5'STRT-seq method was introduced in 2017. The STRT-seq-2i employs a dual index to allow multiplexing and 9600 microwell array capture. This method also limits dilution and eliminates the need for FACS singlecell isolation with an imaging checkpoint (26), providing an ideal platform for high throughput experiments at relatively low cost, without compromising sensitivity and accuracy.

Recent development of droplet-based, ultra-high-throughput scRNA-seq platforms, including 10X Chromium, inDrop, and Drop-seq, enable large-scale scRNA-seq studies, such as Tabula Muris, a compendium of scRNA-seq data, as mentioned above $(18,27)$. A systematic comparison of these three widely used platforms has recently been done (28). In brief, all three methods share $3^{\prime}$ end bias mRNA digital counting, and the barcoded primer beads share a common structure, containing a PCR handle, cell barcode, UMI, and poly-T, except that inDrop beads also have a T7 promoter and a photo-cleavable moiety. Drop-seq uses small, hard resin beads, whereas both 10X and inDrop use soft and deformable hydrogel beads, which enable encapsulation to achieve a super-Poisson distribution and double Poisson distribution for Drop-seq. Thus, the cell-capture efficiency is markedly higher in the 10X and inDrop approaches (29-31). Systematic comparison showed that 10X Chromium outperforms Drop-seq and inDrop, in terms of higher molecular sensitivity and decreased technical noise (32, 33). However, high performance comes with high costs. The cost per cell is around $\$ 0.50$ for $10 \mathrm{X}$ Chromium and requires a Chromium controller. In comparison with compromises in sensitivity and precision, Drop-seq costs $\$ 0.10$ per cell and inDrop cost $\$ 0.25$ (28).

An automated massively parallel scRNA-seq (MARS-seq) approach was introduced in 2014. This approach is based on FACS of individual cells into 384-well plates and uses three levels of barcoding (molecular, cellular, and plate-level tags) thus facilitates molecule counting with a high degree of multiplexing (34). The second generation of MARS-seq 2.0 was published in 2019. In this generation they used longer cell barcodes (from $6 \mathrm{bp}$ to $7 \mathrm{bp}$ ) and UMls (from $4 \mathrm{bp}$ to $8 \mathrm{bp}$ ), which have improved throughput, robustness, and reduced noise and costs (35).

An ideal platform should combine high throughput capabilities with flexibility in sample size to allow for profiling of increasing numbers of cells and multiple samples in parallel and identify new or rare subpopulations while maintaining sensitivity and robustness. Additional desirable features include imaging software to identify contaminating doublets and measure fluorescent reporters. Although the aforementioned methods meet most of these requirements, they are often ex- pensive and require customized equipment, microfluidics, microwells, or cell sorters. Droplet microfluidics allow for high throughput methods at a low cost per cell, however this comes at the expense of flexibility and imaging. A more scalable single-cell profiling method with no need of customized equipment, split-pool ligation-based transcriptome sequencing (SPLiTseq) was reported in 2018 (36). This method is compatible with both fixed cells and nuclei, allowing efficient sample multiplexing and library preparation using only basic laboratory equipment (36).

Although recent advances in scRNA-seq techniques have led to increased use of the technology, it is important to recognize the limitations of single-cell isolation including the loss of spatial information. This limitation was overcome by researchers in 2016, when ST was developed (10). The ideas behind this technique were simple; they introduced positional molecular barcodes and synthesized cDNA on a surface of the tissue sections and were captured for downstream next-generation sequencing. This technique can capture mRNA from tissues with minimal diffusion and maintain positional representation of these mRNAs (10). The barcoded reads can then be overlayed on the tissue section images. Although this method gives a single, spatially resolved whole-transcriptome read out, the resolution of barcoded regions is limited $100 \mu \mathrm{m}$ in diameter, which is not at the level of single cell. ST was further developed, and both resolution (from $100 \mu \mathrm{m}$ to $50 \mu \mathrm{m}$ ) and processing time were improved by $10 \mathrm{X}$ Genomics and were available under the name "10X Visium" $(12,37)$.

A higher spatial-resolution technique called Slide-seq was introduced in 2019 (12), and an improved second generation was described earlier this year (Slide-seq2) (38). Stickels et al. report that modification of barcoded bead synthesis, the sequencing pipeline, and the cDNA-processing steps have markedly improved the mRNA capture sensitivity and efficiency, approaching the level of droplet-based scRNA-seq techniques (38). For further information on the early history and currently availability of ST methods and their applications have been previously reviewed (37).

\section{MAJOR USE OF SCRNA-SEQ ON CARDIOVASCULAR DEVELOPMENT AND DISEASE}

Identification of cell types in tissue/organs and subpopulations of the same cell type

The scRNA-seq studies of the mammalian heart enable researchers to profile a wide range of tissues and cell types in the cardiovascular system. With advances in the resolution of scRNA-seq technologies, novel cell types of the heart have been identified and reported, along with the spatial and temporal expression patterns of various cell types along with the heterogeneity within these subpopulations of cells.

The scRNA-seq method has been used to identify mechanisms of how early cardiac cells emerge and segregate from the heart. As cells develop, their expression patterns change to 
fulfill specific functions, and the expression of these specific genes mark a mature, differentiated phenotype. One can use scRNA-seq to help understand these transitional states and elucidate the activation and deactivation of specific genes that must occur in order for cells to change their profile and identity (39). In the heart, this occurs in the premature venous cells that develop an immature network of vessels, or plexus, during the development of the heart. As these cells continue to mature, some change states by downregulating specific genes, such as CoupTF2 and Nr2f2, and upregulating other genes, such as EphrinB2, DIl4, and Notch4, which ultimately results in reprogramming of cells towards arterial identity (40).

For example, in the postnatal murine heart, valve interstitial cells cluster into two distinct subpopulations at P7 and continue to differentiate into four distinct clusters by P30, undergoing changes in the expression pattern of collagen and elastin and completing morphogenesis of the heart valves (41). In addition to capturing valve interstitial cells, Hulin et al. describe how endothelial-cell and immune-cell populations present at both P7 and P30 remain unchanged in their expression pattern over the course of valve stratification (41).

\section{Cell differentiation and cell lineage analysis in cardiac development}

Using scRNA-seq to analyze the cells that constitute different regions of the heart has also allowed for insight into cell signaling, gene expression patterns and signaling gradients that contribute to the development and physiology of the heart. Employing scRNA-seq in murine embryonic hearts, Li et al. suggest that the expression of Tgf $\beta 1$ by the endocardium and Rspo1 by the epicardium may be implicated in establishing the trabecular myocardium and compact myocardium regions of the developing heart (39). Therefore, the spatial orientation of cells within the heart can affect the profile of a specific cell, and this information can be captured in scRNA-seq data. It is important to keep in mind that the cells that make up the clusters identified by means of scRNA-seq are heterogeneous, themselves, and the signaling that each individual cell receives will affect its individual expression profile.

The scRNA-seq technique has been used to reveal pathogenic mechanisms of congenital heart disease by defining local changes of subpopulations. Soysa et al. used scRNA-seq with a network-based computational method to predict lineage-specifying transcription factors (42). Hand2 was specified as a biomarker for right ventricular formation during early cardiac development that was responsible for congenital heart disease. Soysa et al. found that Hand2 led to dysregulation of retinoicacid signaling and disruption of anterior-posterior patterning by using temporal single-cell-transcriptome analysis and in situ hybridization (42). These findings showed that scRNA-seq is critical for understanding pathological mechanisms of congenital heart disease in a single-cell resolution; it is expected that scRNA-seq can be applied to identify various diseases that have been caused by heterogeneous cell differentiation (11).

\section{Adult heart and cardiovascular disease models from a single-cell perspective}

Similar to the use of scRNA-seq during development, the technique is also used for characterizing cell subpopulations, including rare cell type that constitute adult tissues. This enables researchers to elucidate pathological conditions or celltype, and determine the cell trajectory in injury or disease by characterizing changes in gene expression, signaling pathways, and interaction networks in the adult cardiac cells.

The identification of different subpopulations and rare cell types has major clinical implication for cardiovascular pathogenesis. For example, Skelly et al. identified hybrid macrophage/ fibroblast subpopulations of cells in the mouse heart that may contribute to myocardial fibrosis upon stress (43). But the exact underlying mechanism of fibrosis has been unknown. To access IL-11's role in cardiac fibrosis development, Schafer et al. performed bulk scRNA-seq in the fibrotic human heart and found that IL-11 RNA expression was positively correlated with myofibroblast populations. Additional scRNA-seq experiments in a fibrosis-susceptible mouse model identified a IL-11-positive subpopulation of fibroblast that shows IL-11's important role in myofibroblast differentiation from fibroblasts in cardiovascular disease (44).

The scRNA-seq technique has found greater heterogeneity across these cell types that was not determined from bulk RNA-seq. See et al. also discovered a heterogeneous cardiomyocytes subpopulation from both human and mouse cardiac failure that expresses non-coding RNAs that regulate the cardiomyocyte cell cycle under myocardial stress (45).

Additionally, Nomura et al. use SMART-seq2-based scRNAseq to identify heterogeneous gene expressions of hypertrophic cardiomyocytes and cellular-trajectory analyses of cardiomyocytes at different timepoints from a hypertrophic cardiomyocyte model that was induced by transverse aortic constriction, this revealed the role of p53 in promoting a pathogenic gene program in a subpopulation of cardiomyocytes (46). Additional scRNA-seq data confirmed this finding from patients with cardiac failure. In addition, Nomura et al. used a cell-specific marker, such as a smFISH of RNA molecules, into the scRNA-seq pipeline, which is expected to provide novel biomarkers for disease analysis with a spatial map of cells-ofinterest $(46,47)$.

\section{Single-cell maps of the human heart using ST}

A major hurdle of transcriptome-wide scRNA-seq studies is that they don't retain spatial information of the original cells, because scRNA-seq analysis processing involves dissociation of a tissue into hundreds or thousands of individual cells, each of which is analyzed to define its gene-expression profile (48). Without local information on individual cells, accurate interpretation and application of the data can be difficult $(37,38)$.

To analyze cardiac gene-expression patterns and cell heterogeneity simultaneously, Asp et al. designed a molecular approach that uses scRNA-seq along with ST and ISS, that provide posi- 
tional information of cells (37). ST allows for the quantitative spatial distribution of mRNA transcripts using barcoded oligodT arrays in histological sections, which can be validated using ISS, a technique that em rolling circle amplification ploys padlock probes and rolling circle amplification to visualize genes which have known primers (9). In the paper, Asp et al. not only test their technical proof of concept but also uncover novel cell types, such as clusters of fibrosis-associated fibroblast-like cells and a subpopulation of cardiac muscle cells (37). They also visualized their result by integrating the spatial information into 3D transcriptional maps for other researchers. A major caveat of this combined approach is that it maximizes the efficiency of using data information by providing both cell-type and regional information from tissues, e.g., human embryo tissue, that are not easily obtained.

\section{LIMITATIONS AND FUTURE PERSPECTIVES}

Although scRNA-seq technology has become a powerful and common place tool for researchers to understand physiological and pathological mechanisms, technical challenges and limitations still remain (21). As shown in Fig. 1, tissue dissociation is the initial step for the scRNA-seq pipeline; hence an optimal tissue dissociation protocol is essential depending on the tissue of interest. The isolation and handling of individual cells have become much simpler in recent years. However, a limitation of automated scRNA-seq platforms is the requirement for large volumes of cells compared to those needed to perform manual scRNA-seq methods. In principle, this can be overcome by expanding the pool of barcoded beads and reducing the sample capture speed, with cost timeliness as a tradeoff. Most importantly, only 5-20\% transcripts are typically captured per cell; any events or conditions that may induce stress and influence the transcriptomes need to be addressed and optimized. This issue can be partially addressed by adjusting the depth of sequencing but again, increase cost.

As innovation and advancement in scRNA-seq technologies continues, deciding which scRNA-seq technique is most conducive towards addressing the goals of the study should include analysis of the benefits and drawbacks of different sequencing platforms. Currently, scRNA-seq techniques and the fundamental differences in their approach to obtain single-cell transcripts are summarized in Table 1. There is no one-size fits all; each technique has method-specific advantages and limitations. Hence researchers should choose a technique that aligns with their desired goals and sample availability. Regardless of these technical limitations, single-cell analysis has revolutionized our understanding of cardiovascular development and disease. The applications and insights that in silico analysis enable in the investigation of cell subpopulation variants and differentiation in cardiovascular disease and fetal heart development are invaluable to both basic and medical research and scRNAseq technologies are expected to continue to develop rapidly with continuous improvement of experimental and analytical methods.

\section{ACKNOWLEDGEMENTS}

This work was supported by the Ministry of Health \& Welfare (HI18C2383) and the Ministry of Trade, Industry and Energy (N0002310) to J.K., Republic of Korea.

\section{CONFLICTS OF INTEREST}

The authors have no conflicting interests.

\section{REFERENCES}

1. Lipsett DB, Frisk M, Aronsen JM et al (2019) Cardiomyocyte substructure reverts to an immature phenotype during heart failure. J Physiol 597, 1833-1853

2. Eberwine J, Sul JY, Bartfai T and Kim J (2014) The promise of single-cell sequencing. Nat Methods 11, 25-27

3. Olsen TK, Baryawno N (2018) Introduction to single-cell RNA sequencing. Curr Protoc Mol Biol 122, 1-14

4. Tang F, Barbacioru C, Wang Y et al (2009) mRNA-Seq whole-transcriptome analysis of a single cell. Nat Methods 6, 377-382

5. Chen G, Ning B and Shi T (2019) Single-cell RNA-seq technologies and related computational data analysis. Front Genet 10, 1-13

6. Ziegenhain C, Vieth B, Parekh S et al (2017) Comparative analysis of single-cell RNA sequencing methods. Mol Cell 65, 631-643 e634

7. Ramsköld D, Luo S, Wang YC et al (2012) Full-length mRNA-Seq from single-cell levels of RNA and individual circulating tumor cells. Nat Biotechnol 30, 777-782

8. Sasagawa Y, Nikaido I, Hayashi T et al (2013) Quartz-Seq: A highly reproducible and sensitive single-cell RNA sequencing method, reveals nongenetic gene-expression heterogeneity. Genome Biol 14, 1-17

9. Qian X, Harris KD, Hauling T et al (2020) Probabilistic cell typing enables fine mapping of closely related cell types in situ. Nat Methods 17, 101-106

10. Ståhl PL, Salmén F, Vickovic S et al (2016) Visualization and analysis of gene expression in tissue sections by spatial transcriptomics. Science 353, 78-82

11. Tang X, Huang Y, Lei J, Luo H, Zhu X (2019) The single-cell sequencing: New developments and medical applications. Cell Biosci 9, 1-9

12. Rodriques SG, Stickels RR, Goeva A et al (2019) Slide-seq: A scalable technology for measuring genome-wide expression at high spatial resolution. Science 363, 14631467

13. Kulkarni A, Anderson AG, Merullo DP and Konopka G (2019) Beyond bulk: a review of single cell transcriptomics methodologies and applications. Curr Opin Biotechnol 58, 129-136

14. Choi YH and Kim JK (2019) Dissecting cellular heterogeneity using single-cell RNA sequencing. Mol Cells 42, 189-199

15. Kester L and Oudenaarden AV (2018) Single-cell tran- 
scriptomics meets lineage tracing. Cell Stem Cell 23, 166179

16. Paik DT, Cho S, Tian L, Chang HY and Wu JC (2020) Single-cell RNA sequencing in cardiovascular development, disease and medicine. Nat Rev Cardiol [Online ahead of print]

17. Eberwine J, Yeh $\mathrm{H}$, Miyashiro K et al (1992) Analysis of gene expression in single live neurons. Proc Natl Acad Sci U S A 89, 3010-3014

18. Schaum N, Karkanias J, Neff NF et al (2018) Single-cell transcriptomics of 20 mouse organs creates a Tabula Muris. Nature 562, 367-372

19. Islam S, Kjällquist U, Moliner A et al (2012) Highly multiplexed and strand-specific single-cell RNA $5^{\prime}$ end sequencing. Nat Protoc 7, 813-828

20. Pollen AA, Nowakowski TJ, Shuga J et al (2014) Lowcoverage single-cell mRNA sequencing reveals cellular heterogeneity and activated signaling pathways in developing cerebral cortex. Nat Biotechnol 32, 1053-1058

21. Svensson V, Vento-Tormo R and Teichmann SA (2018) Teichmann, exponential scaling of single-cell RNA-seq in the past decade. Nat Protoc 13, 599-604

22. Picelli S, Björklund ÅK, Faridani OR, Sagasser S, Winberg G and Sandberg R (2013) Smart-seq2 for sensitive full-length transcriptome profiling in single cells. Nat Methods 10, 1096-1100

23. Picelli S, Faridani OR, Björklund AK, Winberg G, Sagasser $S$ and Sandberg R (2014) Full-length RNA-seq from single cells using Smart-seq2. Nat Protoc 9, 171-181

24. Hashimshony T, Wagner F, Sher N and Yanai I (2012) CEL-Seq: Single-cell RNA-seq by multiplexed linear amplification. Cell Rep 2, 666-673

25. Hashimshony T, Senderovich N, Avital G et al (2016) CEL-Seq2: Sensitive highly-multiplexed single-cell RNA-Seq. Genome Biol 17, 1-7

26. Hochgerner $H$, Lönnerberg $P$, Hodge $R$ et al (2017) STRTseq-2i: Dual-index $5^{\prime}$ single cell and nucleus RNA-seq on an addressable microwell array. Sci Rep 7, 1-8

27. Klein AM, Mazutis L, Akartuna I et al (2015) Droplet barcoding for single-cell transcriptomics applied to embryonic stem cells. Cell 161, 1187-1201

28. Zhang X, Li T, Liu F et al (2019) Comparative analysis of droplet-based ultra-high-throughput single-cell RNA-seq systems. Mol Cell 73, 130-142 e135

29. Goldstein LD, Chen YJ, Dunne J et al (2017) Massively parallel nanowell-based single-cell gene expression profiling. BMC Genomics 18, 1-10

30. Zhang X, Li T, Liu F et al (2019) Comparative analysis of droplet-based ultra-high-throughput single-cell RNA-seq systems. Mol Cell 73, 130-142.e135

31. Macosko EZ, Basu A, Satija R et al (2015) Highly parallel genome-wide expression profiling of individual cells using nanoliter droplets. Cell 161, 1202-1214

32. Habib N, Avraham-Davidi I, Basu A et al (2017) Massively parallel single-nucleus RNA-seq with DroNc-seq. Nat
Methods 14, 955-958

33. Zheng GXY, Terry JM, Belgrader P et al (2017) Massively parallel digital transcriptional profiling of single cells. Nat Commun 8, 14049

34. Jaitin DA, Kenigsberg E, Keren-Shaul H et al (2014) Massively parallel single-cell RNA-seq for marker-free decomposition of tissues into cell types. Science 343, 776-779

35. Keren-Shaul H, Kenigsberg E, Jaitin DA et al (2019) MARSseq2.0: an experimental and analytical pipeline for indexed sorting combined with single-cell RNA sequencing. Nat Protoc 14, 1841-1862

36. Rosenberg $A B$, Roco CM, Muscat RA et al (2018) Single-cell profiling of the developing mouse brain and spinal cord with split-pool barcoding. Science 360, 176-182

37. Asp M, Bergenstråhle J and Lundeberg J (2020) Spatially resolved transcriptomes - next generation tools for tissue exploration. Bioessays e1900221 [Online ahead of print]

38. Stickels RR, Murray E, Kumar P et al (2020) Sensitive spatial genome wide expression profiling at cellular resolution. bioRxiv https://doi.org/10.1101/2020.03.12.989806

39. Li G, Tian L, Goodyer W et al (2019) Single cell expression analysis reveals anatomical and cell cycle-dependent transcriptional shifts during heart development. Development 146, dev173476

40. Su T, Stanley G, Sinha R et al (2018) Single-cell analysis of early progenitor cells that build coronary arteries. Nature 559, 356-362

41. Hulin A, Hortells L, Gomez-Stallons MV et al (2019) Maturation of heart valve cell populations during postnatal remodeling. Development 146, dev173047

42. Soysa TY, Ranade SS, Okawa S et al (2019) Single-cell analysis of cardiogenesis reveals basis for organ-level developmental defects. Nature 572, 120-124

43. Skelly DA, Squiers GT, McLellan MA et al (2018) Singlecell transcriptional profiling reveals cellular diversity and intercommunication in the mouse heart. Cell Rep 22, 600610

44. Schafer S, Viswanathan S, Widjaja AA et al (2017) IL-11 is a crucial determinant of cardiovascular fibrosis. Nature $552,110-115$

45. See Kelvin, Tan WLW, Lim EH et al (2017) Single cardiomyocyte nuclear transcriptomes reveal a lincRNA-regulated de-differentiation and cell cycle stress-response in vivo. Nat Commun 8, 225

46. Nomura S, Satoh M, Fujita T et al (2018) Cardiomyocyte gene programs encoding morphological and functional signatures in cardiac hypertrophy and failure. Nat Commun 9, 4435

47. Gaspar I, Ephrussi A (2015) Strength in numbers: quantitative single-molecule RNA detection assays. Wiley Interdiscip Rev Dev Biol 4, 135-150

48. Westoby J, Artemov P, Hemberg M and Ferguson-Smith A (2020) Obstacles to detecting isoforms using full-length scRNA-seq data. Genome Biol 21, 1-19 